\title{
Ultrasonication-assisted synthesis of $\mathrm{CsPbBr}_{3}$ and $\mathrm{Cs}_{4} \mathrm{PbBr}_{6}$ perovskite nanocrystals and their reversible transformation
}

\author{
Longshi Rao $^{1,2}$, Xinrui Ding ${ }^{*}$, Xuewei Du ${ }^{1}$, Guanwei Liang ${ }^{1}$, Yong Tang ${ }^{1}$, Kairui Tang ${ }^{3}$ \\ and Jin Z. Zhang ${ }^{2}$
}

Open Access

\author{
Full Research Paper \\ Address: \\ ${ }^{1}$ Engineering Research Centre of Green Manufacturing for \\ Energy-Saving and New-Energy Technology, School of Mechanical \\ and Automotive Engineering, South China University of Technology, \\ Guangzhou 510640, China, ${ }^{2}$ Department of Chemistry and \\ Biochemistry, University of California, Santa Cruz, CA 95064, USA \\ and ${ }^{3}$ Mechanical Engineering, Pennsylvania State University, \\ Harrisburg, PA 17057, USA \\ Email: \\ Xinrui Ding* - dingxr@scut.edu.cn \\ * Corresponding author \\ Keywords: \\ $\mathrm{CsPbBr}_{3} \mathrm{PNCs} ; \mathrm{Cs}_{4} \mathrm{PbBr}_{6}$ PNCs; polar-solvent-free; reversible \\ transformation; ultrasonication
}

Beilstein J. Nanotechnol. 2019, 10, 666-676. doi:10.3762/bjnano.10.66

Received: 31 October 2018

Accepted: 13 February 2019

Published: 06 March 2019

This article is part of the thematic issue "Low-dimensional materials and systems".

Guest Editor: S. Walia

(C) 2019 Rao et al.; licensee Beilstein-Institut.

License and terms: see end of document.

\begin{abstract}
We demonstrate an ultrasonication-assisted synthesis without polar solvent of $\mathrm{CsPbBr}_{3}$ and $\mathrm{Cs}_{4} \mathrm{PbBr}_{6}$ perovskite nanocrystals (PNCs) and their reversible transformation. The as-prepared $\mathrm{CsPbr}_{3} \mathrm{PNCs}$ and $\mathrm{Cs}_{4} \mathrm{PbBr}_{6} \mathrm{PNCs}$ exhibit different optical properties that depend on their morphology, size, and structure. The photoluminescence (PL) emission and quantum yield (QY) of the $\mathrm{CsPbr}_{3} \mathrm{PNCs}_{\mathrm{P}}$ can be tuned by changing the ultrasound power, radiation time, and the height of the vibrating spear. The optimized $\mathrm{CsPbr}_{3} \mathrm{PNCs}$ show a good stability and high PL QY of up to $85 \%$. In addition, the phase transformation between $\mathrm{CsPbr}_{3} \mathrm{PNCs}$ and $\mathrm{Cs}_{4} \mathrm{PbBr}_{6} \mathrm{PNCs}$ can be obtained through varying the amount of oleylamine $(\mathrm{OAm})$ and water. The mechanism of this transformation between the $\mathrm{CsPbBr}_{3} \mathrm{PNCs}_{\text {and }} \mathrm{Cs}_{4} \mathrm{PbBr}_{6} \mathrm{PNCs}$ and their morphology change are studied, involving ions equilibrium, anisotropic growth kinetics, and $\mathrm{CsBr}$-stripping process.
\end{abstract}

\section{Introduction}

Metal halide perovskite nanocrystals (PNCs) are promising candidates for application in the fields of light-emitting diodes (LEDs) [1,2], high-efficiency solar cells [3], low-threshold lasers [4], and photodetectors [5]. Compared to traditional semiconductors, colloidal PNCs demonstrate excellent properites, such as tunable photoluminescence (PL) throughout the visible spectrum, super high PL quantum yield (QY), low trap-state density, and narrow emission linewidth [6-8]. The crystal structure of $\mathrm{CsPbX}_{3}\left(\mathrm{X}=\mathrm{Cl}^{-}, \mathrm{Br}^{-}, \mathrm{I}^{-}\right)$PNCs consists of a 12-fold coordinated network created by $\left[\mathrm{PbX}_{6}\right]^{4-}$ octahedra in which the $\mathrm{Cs}^{+}$ions reside in the periphery of this network $[9,10]$. These PNCs are prone to structural instabilities and phase trans- 
formations involving ion migration and interface hydration [11]. However, this phase and structure versatility has become the great advantage of PNCs in their technical applications, especially in optoelectronics. Although the focus has been on the $\mathrm{CsPbX}_{3}$ structure, researchers start to turn their attention on synthesizing new perovskite materials, such as $\mathrm{Cs}_{4} \mathrm{PbX}_{6}$ PNCs. Under Cs-rich or Pb-poor synthesis conditions, zero-dimensional (0D) structures of $\mathrm{Cs}_{4} \mathrm{PbX}_{6}$ NCs can be achieved, demonstrating a crystalline structure with well-separated octahedra $\left[\mathrm{PbBr}_{6}\right]^{4-}$ isolated by $\mathrm{Cs}^{+}$ions $[12,13]$. This specific structure is expected to result in strong quantum confinement and electron-phonon interactions. This inspires researchers to further explore this structure. The key to this exploration is the development of various approaches to the synthesis of highquality PNCs.

Since, in 2015, Kovalenko and co-workers synthesized $\mathrm{CsPbX}_{3}$ PNCs by using a hot-injection method, great successes in the controlled synthesis and application of the $\mathrm{CsPbX}_{3}$ PNCs have been achieved in a very short time [14]. To date, the most commonly adopted approach for synthesizing highly efficient PNCs are solution-based procedures, including hot injection, solvothermal synthesis, microreactor synthesis, and room-temperature (RT) ligand-mediated reprecipitation, in which shape and size are tuned through the control of temperature, reaction time, and composition of the precursors [15-17]. Chen et al. demonstrated a facile solvothermal method for preparing $\mathrm{CsPbX}_{3}$ PNCs with adjustable optical properties [18]. Additionally, Li's group reported a poly(lactic acid)-assisted anion-exchange method using a microreactor for tuning the emission spectra of $\mathrm{CsPbX}_{3}$ PNCs from green to near-ultraviolet, which might be applicable for mass production [19]. Besides, great efforts have been made to prepare PNCs through the chemical transformation of pre-synthesized PNCs [20-22]. For example, Wu et al. reported a CsX-stripping method that enabled the transformation of nonluminescent $\mathrm{Cs}_{4} \mathrm{PbX}_{6}$ PNCs to highly luminescent $\mathrm{CsPbX}_{3}$ PNCs through an interfacial reaction [20]. They focus on investigating the water-triggered transformation process between $\mathrm{Cs}_{4} \mathrm{PbX}_{6} \mathrm{PNCs}$ and $\mathrm{CsPbX}_{3}$ PNCs in a different phase. Similar methods were applied to explore new perovskite materials such as $\mathrm{Cs}_{4} \mathrm{PbBr}_{6}$. Zhai et al. showed a simple solvothermal approach for synthesizing $\mathrm{CsPbr}_{3}$ nanoplatelets and their phase transformation to $\mathrm{Cs}_{4} \mathrm{PbBr}_{6} \mathrm{PNCs}$ [23]. Liu and co-workers also demonstrated that $\mathrm{CsPbr}_{3}$ PNCs were successfully converted to $\mathrm{Cs}_{4} \mathrm{PbBr}_{6} \mathrm{PNCs}$ through a "ligand-mediated transformation" method with the addition of oleylamine (OAm) [24]. Udayabhaskararao's group showed the reversible transformation from $\mathrm{CsPbX}_{3}$ to $\mathrm{Cs}_{4} \mathrm{PbX}_{6}$ through the ratio of oleic acid (OA) to OAm in a Brønsted acid-base equilibrium [25]. Despite the progress made in obtaining PNCs, in general, inert conditions, high temperature and pre-synthesized precur- sors are required for hot injection. In addition, RT methods were mostly carried out by mixing a polar solvent with a large amount of nonpolar solvent. Since PNCs are reported to be very sensitive to polar solvents, these methods result in the inevitable degradation of PNCs, especially for iodine-based PNCs [26-28]. Therefore, in order to obtain PNCs with high PL QY and stability, it is crucial to develop synthesis methods free of polarsolvents.

To date, some attempts have been made to synthesize PNCs without the use of polar solvents. Tong's group demonstrated the single-step and polar-solvent-free synthesis of $\mathrm{CsPbX}_{3}$ PNCs with tunable halide ion composition and thickness through the direct ultrasonication of precursors [29]. Whereas this method has been reported for synthesizing PNCs without using polar solvents, it does not allow for a control over dimensionality and phase transformation. We recently reported a fast, low-cost, environmentally friendly, and polar-solvent-free strategy for synthesizing all-inorganic $\mathrm{CsPbBr}_{3} \mathrm{NCs}$ with tunable shape and size [30]. During this process, we found that a great excess of $\mathrm{OAm}$ results in the formation of a derivative of $\mathrm{CsPbBr}_{3} \mathrm{NCs}$, i.e., $\mathrm{Cs}_{4} \mathrm{PbBr}_{6}$. However, the underlying transformation mechanism has not been fully understood. Following this, we set out here to expand this study to control the phase transformation. $\mathrm{CsPbBr}_{3}$ PNCs as precursor were obtained by modifying the approach initially presented by Tong, which was recently elaborated by our group [29,30]. We demonstrated in detail how, by tuning the ultrasound power and time, the PL emission of $\mathrm{CsPbBr}_{3}$ PNCs can be precisely controlled. Benefitting from this knowledge, here we attained $\mathrm{CsPbBr}_{3}$ PNCs with a high PL QY (ca. 85\%) by optimizing the immersion height of the vibrating spear in the liquid. In addition, the phase transformation of $\mathrm{CsPbBr}_{3}$ PNCs to $\mathrm{Cs}_{4} \mathrm{PbBr}_{6}$ PNCs was achieved in this study by direct ultrasonication of solid powders or by adding $\mathrm{OAm}$ in the solution of pre-synthesized $\mathrm{CsPbBr}_{3}$ PNCs. Finally, inspired by the method proposed by $\mathrm{Wu}$ et al. [20], a successful structure conversion from $\mathrm{Cs}_{4} \mathrm{PbBr}_{6}$ PNCs to $\mathrm{CsPbBr}_{3} \mathrm{PNCs}$ was obtained here by simply adding different amounts of water into pre-synthesized $\mathrm{Cs}_{4} \mathrm{PbBr}_{6} \mathrm{PNCs}$. The mechanism behind phase transformation and morphology change were investigated by using a combination of spectroscopy and microscopy techniques.

\section{Results and Discussion Characterization of $\mathrm{CsPbBr}_{3}$ PNCs}

The typical procedure for synthesizing $\mathrm{CsPbBr}_{3}$ and $\mathrm{Cs}_{4} \mathrm{PbBr}_{6}$ PNCs and for reversibly transforming them is illustrated in Scheme 1. $\mathrm{Cs}_{2} \mathrm{CO}_{3}$ and $\mathrm{PbBr}_{2}$ were loaded into the liquid paraffin/OAm/OA solution. Then, the precursors were processed by tip-sonication and purified via centrifuging in the presence of methyl acetate as precipitation agent. Subsequently, 


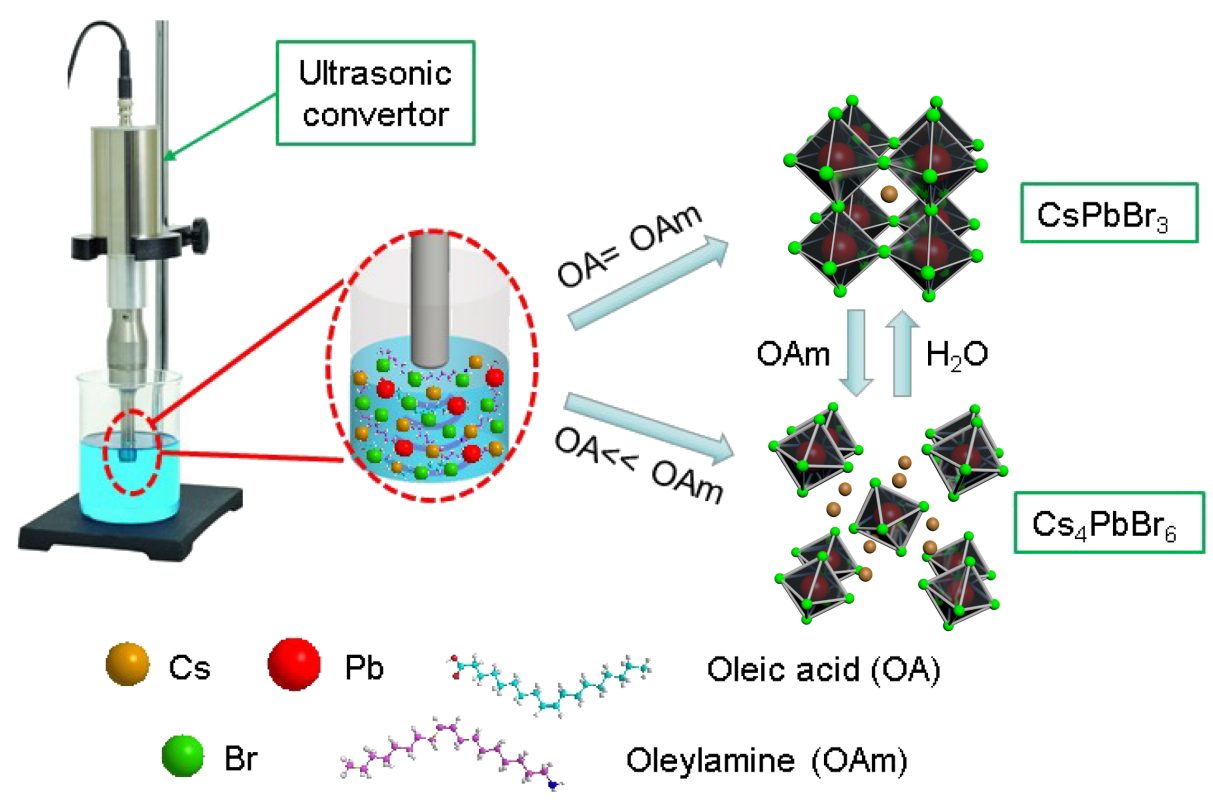

Scheme 1: Illustration of synthesizing $\mathrm{CsPbBr}_{3}$ and $\mathrm{Cs}_{4} \mathrm{PbBr}_{6} \mathrm{PNCs}$ without polar solvent using ultrasonication assistance, and their reversible transformation by adding OAm and water.

the sediment was redispersed in toluene for further characterization. The reversible transformation between $\mathrm{Cs}_{4} \mathrm{PbBr}_{6}$ PNCs and $\mathrm{CsPbBr}_{3}$ PNCs was achieved by changing the amounts of OAm and water. Detailed synthesis conditions are given in the Experimental section.
The crystal structure and morphology of the as-prepared samples were determined by XRD and TEM. As shown in Figure $1 \mathrm{a}$, the diffraction pattern clearly indicates that orthorhombic $\mathrm{CsPbBr}_{3}$ PNCs (PDF card \#18-0364) were formed. No other phases were observed, suggesting the high

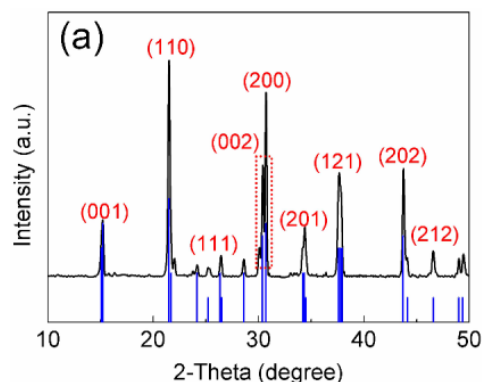

(d)

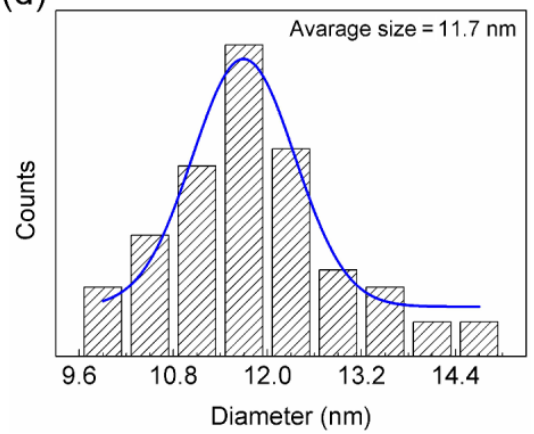

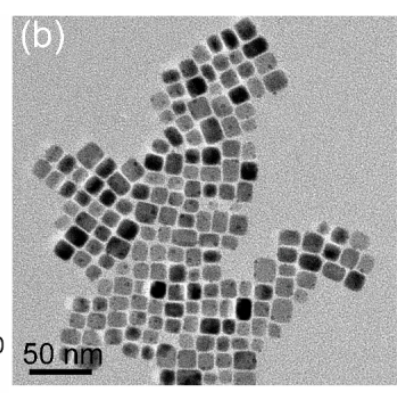

(e)

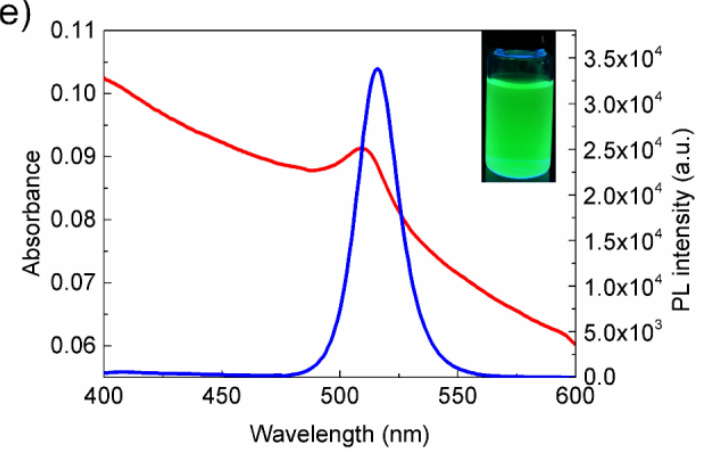

Figure 1: Characterization of the $\mathrm{CsPbBr}_{3} \mathrm{PNCs}$ prepared using ultrasonication assistance. (a) XRD patterns. Black line and blue line represent experimental data and standard reference, respectively. (b) TEM image. (c) HRTEM image. (d) Size distribution. (e) UV-vis absorption spectrum (red line) and PL emission spectrum (blue line). Inset is a photograph under $365 \mathrm{~nm}$ UV irradiation. 
purity of the samples. The TEM image shown in Figure $1 \mathrm{~b}$ demonstrates that the $\mathrm{CsPbBr}_{3}$ PNCs have a regular square morphology. HRTEM was further carried out to measure the lattice spacing of the product. Figure 1c shows a lattice spacing distance of ca. $0.41 \mathrm{~nm}$ for the $\mathrm{CsPbBr}_{3}$ PNCs. The size distribution shown in Figure 1d indicates that the well-dispersed $\mathrm{CsPbr}_{3} \mathrm{PNCs}_{\mathrm{B}}$ have an average diameter of ca. $11.7 \mathrm{~nm}$. To explore the optical properties of colloidal $\mathrm{CsPbBr}_{3} \mathrm{PNCs}_{\text {, }}$ UV-vis absorption spectra and PL emission spectra were recorded. As shown in Figure 1e, the first excitonic absorption peak was located at $510 \mathrm{~nm}$ and the strong PL emission band centered at $516 \mathrm{~nm}$ was observed with a narrow full width at half maximum (FWHM) of $18 \mathrm{~nm}$, indicating a narrow polydispersity of the PNCs obtained by this method. The PL QY of the as-prepared $\mathrm{CsPbBr}_{3}$ PNCs measured to be ca. $85 \%$ (Rhodamin 101 as reference, PL QY is $100 \%$ ) following a previously published report [31]. In addition, Supporting Information File 1, Figure S1 clearly demonstrates the improved photostability and chemical stability of $\mathrm{CsPbBr}_{3}$ PNCs.

\section{Effect of synthesis conditions}

Our previous study has shown that ultrasound power and radiation time have a great influence on the optical properties of the $\mathrm{CsPbBr}_{3} \mathrm{PNCs}[30]$. In this study, we found that the immersion height of the vibrating spear in the solvent influences the product properties (the effect will be discussed later). We divided the height of liquid into five equal parts, i.e., from the bottom to the surface of the liquid, $1 / 5,2 / 5,3 / 5,4 / 5$, and $5 / 5$.

We first investigated the effect of ultrasound power on the optical properties of $\mathrm{Cs} \mathrm{PbBr}_{3}$ PNCs. To avoid breaking the bottle, the immersion height of the vibrating spear and radiation time are $4 / 5$ and $30 \mathrm{~min}$, respectively. Figure 2a shows the change of UV-vis absorption spectra and PL spectra of the

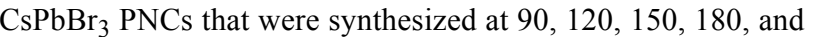
$210 \mathrm{~W}$ of ultrasound power, while keeping other synthesis conditions unchanged. If the ultrasound power is less than $90 \mathrm{~W}$, there is no UV-vis absorption peak and a very weak PL intensity, implying that almost no PNCs formed. However, the first characteristic absorption peak changes to red slowly with an increase of ultrasound power, corresponding to the red-shift of the PL emission peak, which is similar to the findings we recently reported [30]. While higher ultrasound power supports faster dissolution, it has also a strong impact on the homogeneity of the PNCs. For example, when the ultrasound power is $210 \mathrm{~W}$, the UV-vis absorption at long wavelengths is very high, indicating large crystals were formed with strong scattering. Therefore, it is necessary to choose the appropriate ultra- (a)

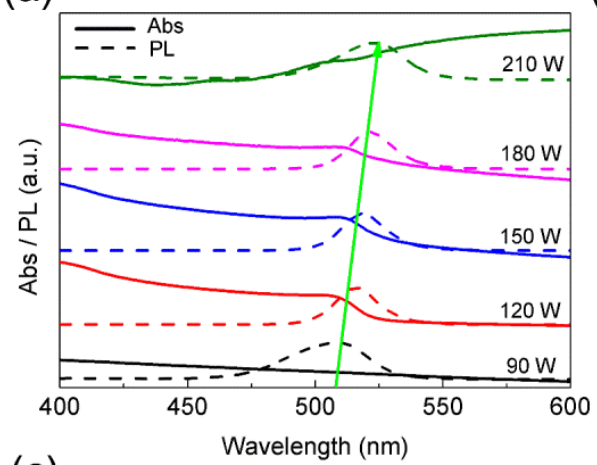

(c)

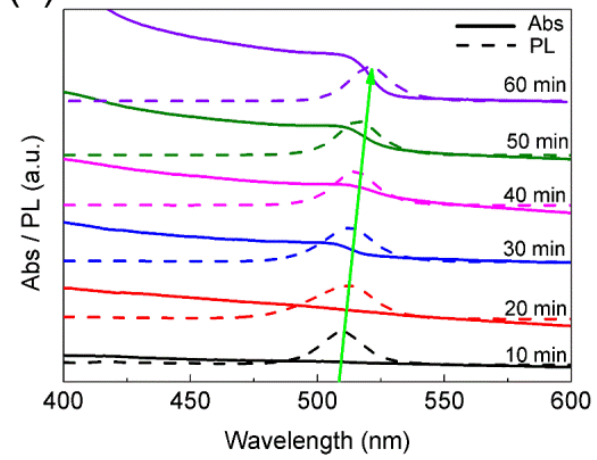

(b)

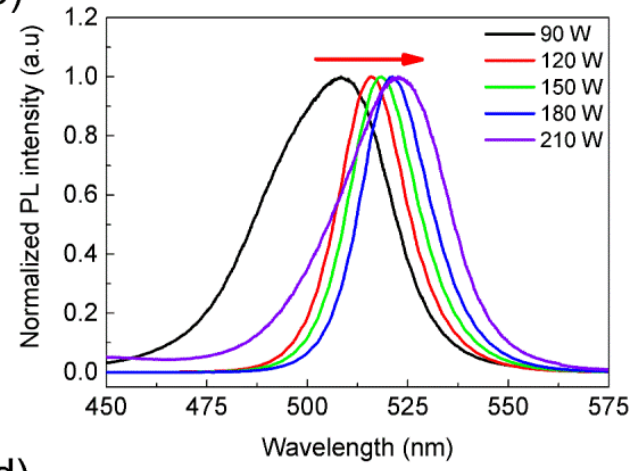

(d)

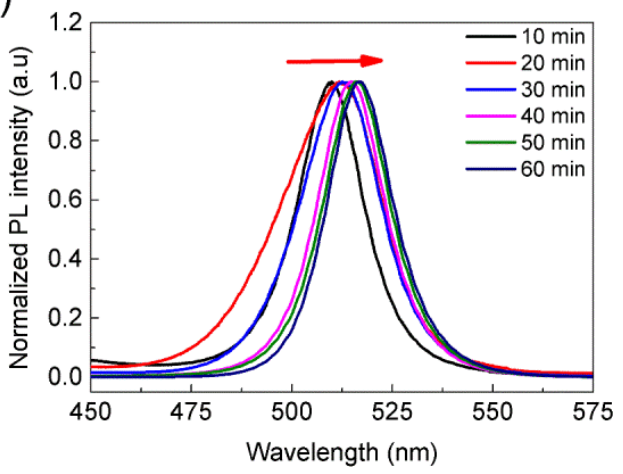

Figure 2: (a) UV-vis absorption spectra and PL emission spectra, and (b) normalized PL emission spectra of the $\mathrm{CsPbBr}_{3} \mathrm{PNCs}$ that were synthesized at $90,120,150,180$, and $210 \mathrm{~W}$ of ultrasound power, respectively. (c) UV-vis absorption spectra and PL spectra, and (d) normalized PL emission spectra of $\mathrm{CsPbBr}_{3}$ PNCs that were synthesized at 10, 20,30, 40, 50, and 60 min of radiation time. 
sound power. The normalized PL emission peaks in Figure $2 b$ shift from 505 to $523 \mathrm{~nm}$, indicating that our approach can precisely modulate PL emission.

Furthermore, we studied the influence of radiation time on the

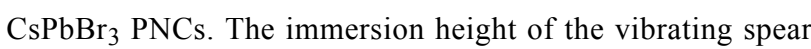
and ultrasound power are $4 / 5$ and $120 \mathrm{~W}$, respectively. As shown in Figure 2c and Figure 2d, when the radiation time is increased, both the UV-vis absorption and PL spectra are redshifted, we suggest that it is the size effect that is dominant over ionic bond strength in causing the spectral shift, which is different from the effects of radiation time that we observed recently [30]. This phenomenon indicates that the immersion height of the vibrating spear would affect PNCs properties.

The effects of the immersion height of the vibrating spear in the liquid were also investigated. The total liquid height was divided into five equal parts as shown in the inset of Figure 3a. Figure 3 a shows the PL intensity (UV-vis absorbance at $400 \mathrm{~nm}$ ) of four samples that were synthesized by setting the immersion height of the vibrating spear to $1 / 5,2 / 5,3 / 5$, and $4 / 5$ of the total liquid height. As the immersion height increases, the corresponding PL intensity obviously decreases. In addition, the $\mathrm{PL} Q Y$ in Figure $3 b$ further confirmed that the $\mathrm{CsPbBr}_{3} \mathrm{PNCs}$ exhibit the best performance when the immersion height of the vibrating spear is set at $1 / 5$ of the total liquid height. Ultrasonication results in a combination of thermal, vibrational, and acoustic cavitation, i.e., the formation, growth, and implosive collapse of bubbles in liquids [32-34]. In the center of these bubbles, extremely high temperatures of about $5000 \mathrm{~K}$ and high pressures of about $20 \mathrm{MPa}$ were achieved by high-intensity ultrasound [32], enabling a quick decomposition of the particles. The lower the immersion height of vibrating spear, the higher temperature and pressure is achieved, which, as a result, benefits the formation of PNCs.

\section{Characterization of $\mathrm{Cs}_{4} \mathrm{PbBr}_{6} \mathrm{PNCs}$}

The current approach can be further used for controlling phase and structure transformations in the PNCs. The method introduced in this work enables the successful synthesis of rhombohedral $\mathrm{Cs}_{4} \mathrm{PbBr}_{6} \mathrm{PNCs}$ via changing the amount of OAm. The amount of OAm was increased to $3.0 \mathrm{~mL}$, while all other conditions were kept the same. The phase of the obtained product was characterized by XRD, as shown in Figure 4a. The XRD pattern with peaks at $2 \theta=12.9,20.1,22.4,25.6,28.630 .3$, $30.9,34.1,39.3$, and $45.7^{\circ}$ correspond to diffractions from (110), (113), (300), (024), (214), (223), (006), (134), (330), and (600) crystal planes of rhombohedral $\mathrm{Cs}_{4} \mathrm{PbBr}_{6}$ (PDF card \#732478) [13]. Meanwhile, weak peaks of $\mathrm{CsPbr}_{3}$ were observed, indicating both $\mathrm{CsPbBr}_{3}$ and $\mathrm{Cs}_{4} \mathrm{PbBr}_{6}$ PNCs were formed during the process.

TEM was further performed to characterize the morphology of as-prepared PNCs. Figure $4 \mathrm{~b}$ shows the formation of $\mathrm{Cs}_{4} \mathrm{PbBr}_{6}$ PNCs with hexagonal crystal structure and confirms the existence of square-shaped $\mathrm{CsPbBr}_{3} \mathrm{PNCs}$. Additionally, Figure 4c shows that the small black spots (green arrows) existing on the surface of the $\mathrm{Cs}_{4} \mathrm{PbBr}_{6}$ PNCs are metallic lead nanoparticles that have been reported before [23,35,36]. The HRTEM image shown in Figure 4d demonstrates an interplanar spacing of $0.39 \mathrm{~nm}$, corresponding to the (300) crystal plane of bulk $\mathrm{Cs}_{4} \mathrm{PbBr}_{6}$, which is also consistent with the PDF card \#73-2478. The size of the $\mathrm{Cs}_{4} \mathrm{PbBr}_{6}$ PNCs is defined here as the longest distance between hexagonal corners, which is ca. $65 \mathrm{~nm}$ for the example shown. Besides, the SEM image illustrates that $\mathrm{Cs}_{4} \mathrm{PbBr}_{6}$ particles are hexagonal prisms with a thickness of ca. $15 \mathrm{~nm}$, as presented in Figure 4d inset and Supporting Information File 1, Figure S2.

The absorption spectrum of the $\mathrm{Cs}_{4} \mathrm{PbBr}_{6} \mathrm{PNCs}$ is very different from that of the $\mathrm{CsPbBr}_{3} \mathrm{PNCs}$, as shown in Figure 4e. The
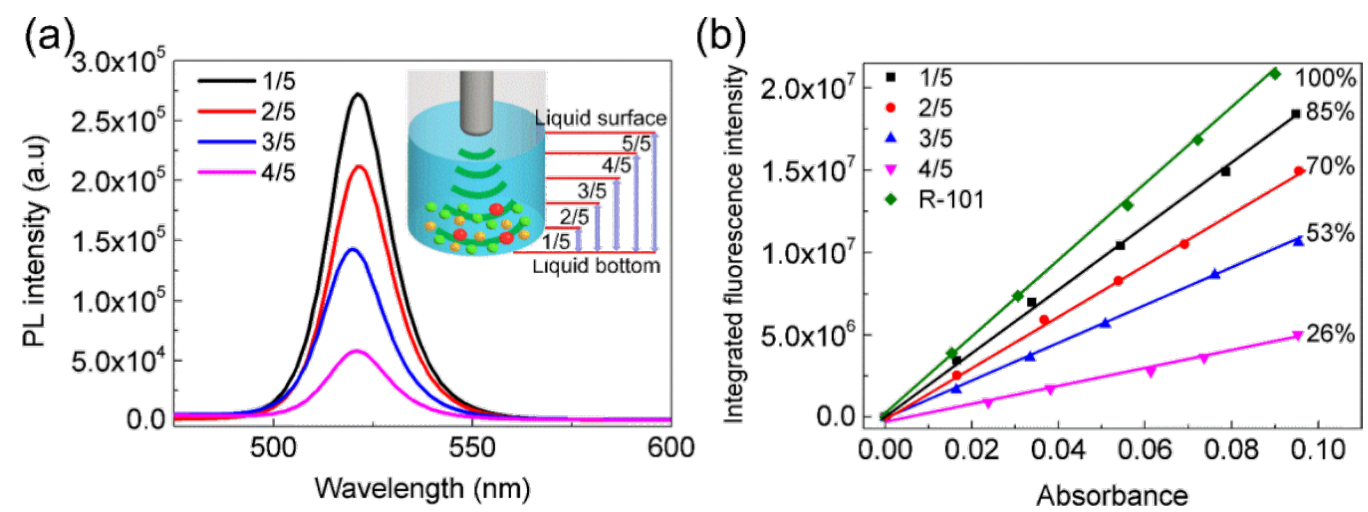

Figure 3: (a) PL emission spectra and (b) PL QY of the CsPbBr 3 PNCs that were synthesized at $1 / 5,2 / 5,3 / 5$, and 4/5 of total liquid height, respectively. $1 / 5,2 / 5,3 / 5,4 / 5$, and $5 / 5$ in (a) inset is one of the five equal parts from liquid bottom to liquid surface. R-101 in (b) is rhodamine 101 (PL QY, $100 \%)$ as the standard sample to test PL QY of PNCs. 

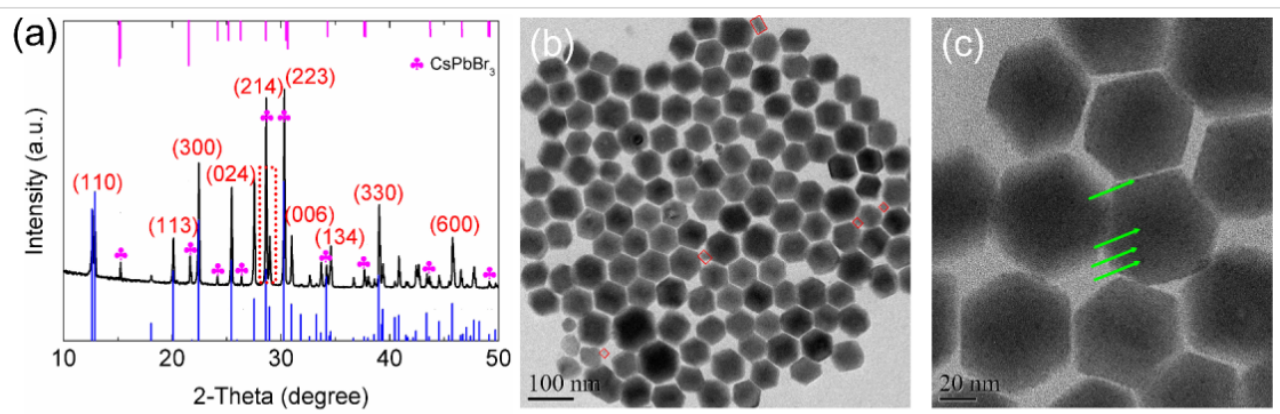

(d)

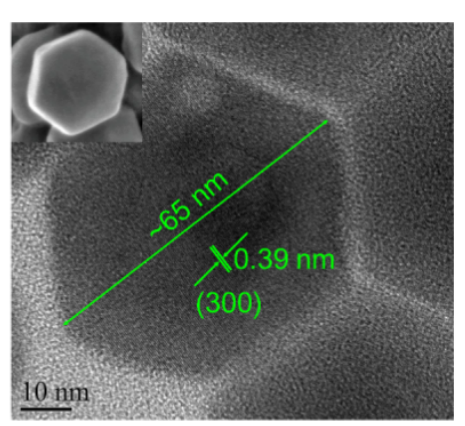

(e)

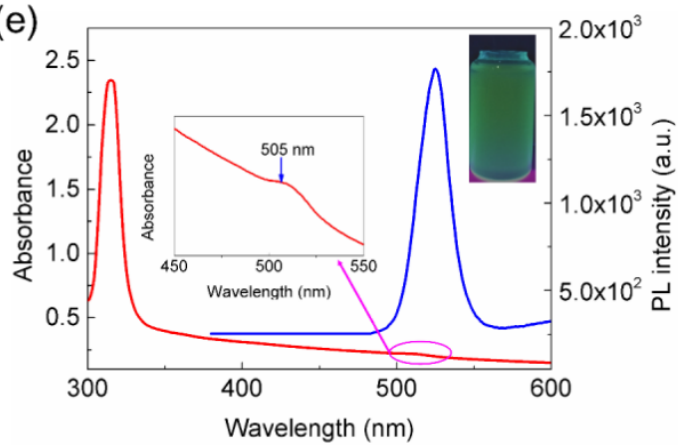

Figure 4: Characterization of the $\mathrm{Cs}_{4} \mathrm{PbBr}_{6} \mathrm{PNCs}$ synthesized using ultrasonication. (a) XRD patterns. Black line represents experimental data; blue line (below) and pink line (upper) represent standard reference bulk $\mathrm{CsPbBr}_{3}$ and $\mathrm{Cs}_{4} \mathrm{PbBr}_{6}$, respectively. (b) TEM image, red squares represent

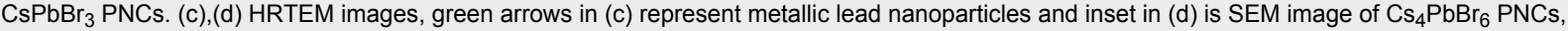
respectively. (e) UV-vis absorption spectra (red line) and PL emission spectra (blue line). Insets are high resolution absorption spectrum and representative digital photograph.

first excitonic absorption has been shifted from $510 \mathrm{~nm}$ for $\mathrm{CsPbBr}_{3} \mathrm{PNCs}$ to $315 \mathrm{~nm}$ for $\mathrm{Cs}_{4} \mathrm{PbBr}_{6}$ PNCs. This absorption feature is consistent with that of bulk $\mathrm{Cs}_{4} \mathrm{PbBr}_{6}$, which was proven to be the localized $6 \mathrm{~s}_{1 / 2}-6 \mathrm{p}_{1 / 2}$ transition within the isolated $\left[\mathrm{PbBr}_{6}\right]^{4-}$ octahedra separated by $\mathrm{Cs}^{+}$ions [24]. A weak characteristic UV-vis absorption and a PL emission peak for $\mathrm{CsPbBr}_{3} \mathrm{PNCs}_{\text {at }} 505 \mathrm{~nm}$ and $520 \mathrm{~nm}$, respectively, further confirmed the existence $\mathrm{CsPbr}_{3}$ PNCs.

In $\mathrm{Cs}_{4} \mathrm{PbBr}_{6} \mathrm{PNCs}$, typically a green emission arises either from defects or from impurities or from a combination of both $[37,38]$. Herein, the purified $\mathrm{Cs}_{4} \mathrm{PbBr}_{6}$ PNCs did not demonstrate $\mathrm{PL}$ emission over the whole visible spectrum due to their wide bandgap $\left(E_{\mathrm{g}}\left(\mathrm{Cs}_{4} \mathrm{PbBr}_{6}\right)=3.94 \mathrm{eV}\right)$, while the observed weak PL emission results from a small portion of $\mathrm{CsPbr}_{3}$ impurities in the $\mathrm{Cs}_{4} \mathrm{PbBr}_{6}$ PNCs (see Figure 3a-e). Since $\mathrm{CsPbBr}_{3} \mathrm{PNCs}$ exhibit a high $\mathrm{PL} \mathrm{QY}$, the green $\mathrm{PL}$ emission is ascribed to minor $\mathrm{CsPbBr}_{3}$ impurities in the samples. This result coincides with previous works on $\mathrm{Cs}_{4} \mathrm{PbBr}_{6}$ PNCs that show a strong green emission at about $500 \mathrm{~nm}$ and confirmed that these green $\mathrm{PL}$ emissions originate from $\mathrm{CsPbBr}_{3} \mathrm{PNC}$ impurities $[10,39,40]$.

Furthermore, the effect of the amount of OAm on the phase transformation was investigated. As shown in Figure 5a, when the amount of OAm ranged from 0.5 to $3.0 \mathrm{~mL}$, the first charac- teristic absorption peak (ca. $510 \mathrm{~nm}$ ) and the PL emission intensity of $\mathrm{CsPbr}_{3} \mathrm{PNCs}$ slowly decrease, while new strong absorption features in the UV region (ca. $315 \mathrm{~nm}$ ) emerge, which have been confirmed to result from the formation of $\mathrm{Cs}_{4} \mathrm{PbBr}_{6}$ PNCs [25]. When adding equal amounts of OAm and $\mathrm{OA}$, there are no other peaks in the UV-vis absorption spectra except for that at ca. $510 \mathrm{~nm}$. With increasing amount of OAm, the absorption intensity at ca. $510 \mathrm{~nm}$ decreases. Simultaneously, the absorption intensity at ca. $315 \mathrm{~nm}$ increases, while the PL intensity decreases (Figure $5 \mathrm{~b}$ ), and is blue-shifted followed by an increase in the FWHM of the PL peak. All these effects suggest the decomposition of the $\mathrm{CsPbr}_{3}$ PNCs. Based on this process it can be concluded that the excess amount of OAm triggers the transformation between $\mathrm{CsPbBr}_{3} \mathrm{PNCs}$ and $\mathrm{Cs}_{4} \mathrm{PbBr}_{6} \mathrm{PNCs}$.

The morphology change from $\mathrm{CsPbBr}_{3}$ PNCs to $\mathrm{Cs}_{4} \mathrm{PbBr}_{6}$ PNCs was further confirmed by using TEM. When the amount of OAm is between 0.5 and $1.5 \mathrm{~mL}$, the morphology of PNCs gradually becomes irregular and some hexagonal shapes emerge (Figure $5 \mathrm{c}-\mathrm{e}$ ). As the amount of OAm increase to $3.0 \mathrm{~mL}$, $\mathrm{Cs}_{4} \mathrm{PbBr}_{6} \mathrm{PNCs}$ with homogeneous hexagonal shape can be achieved (Figure 5h). These results suggest that the growth kinetics of this process can be controlled by adding OAm, and the $\mathrm{PNCs}$ are prone to crystallize in the Cs-rich $\mathrm{Cs}_{4} \mathrm{PbBr}_{6}$ phase when OAm is present in excess. The transformation from 
(a)

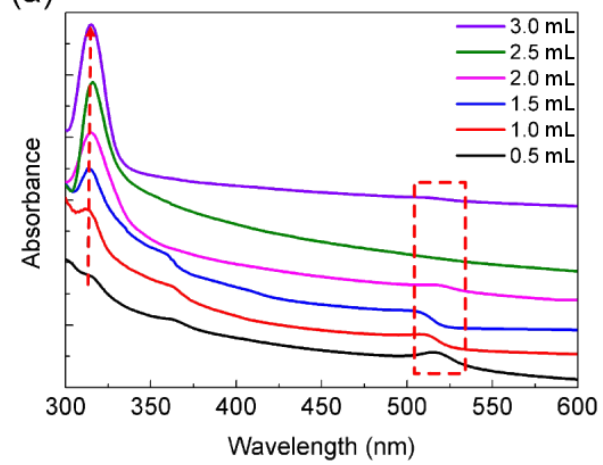

(b)

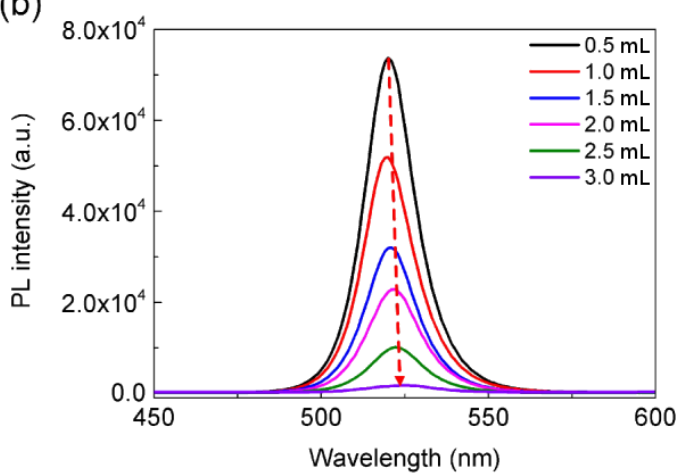

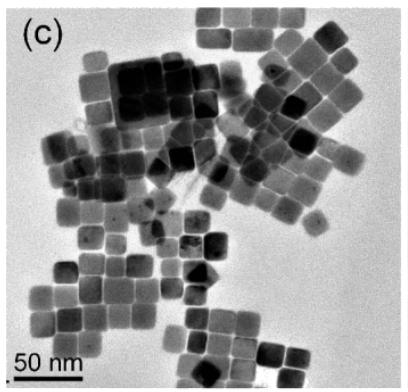
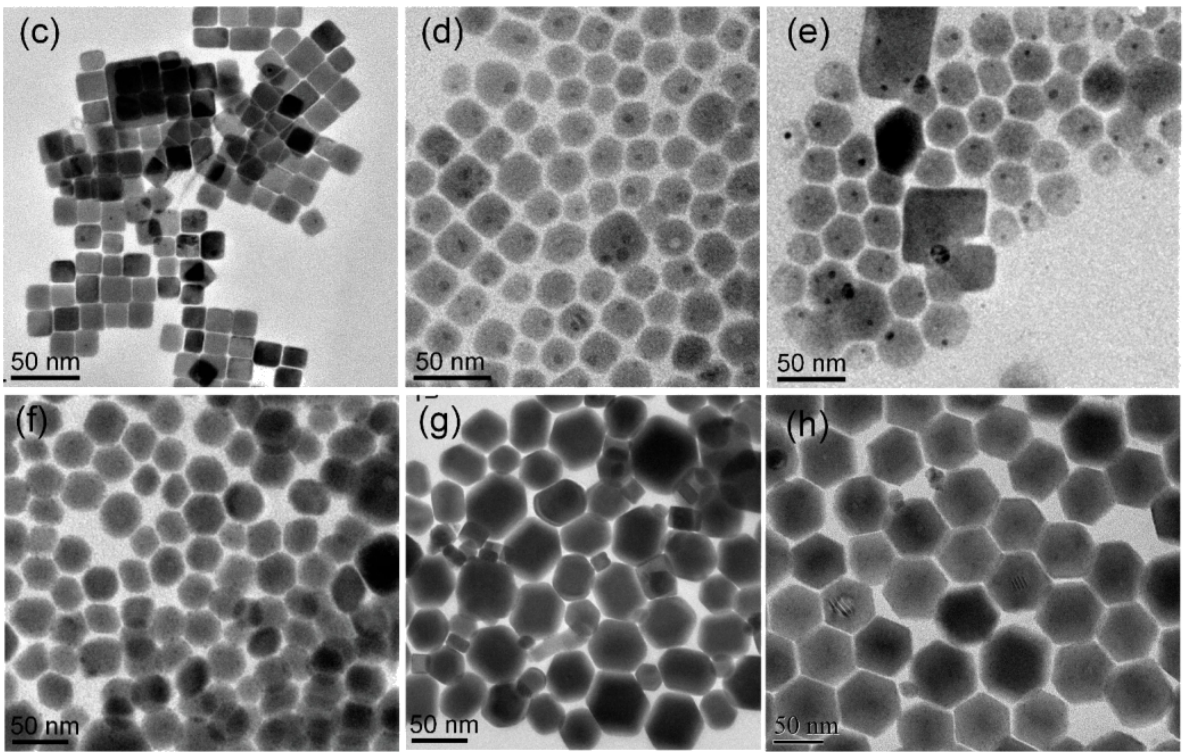

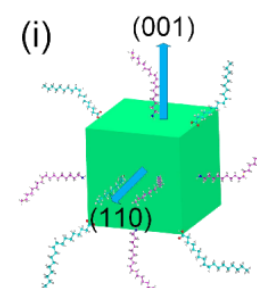

(1)

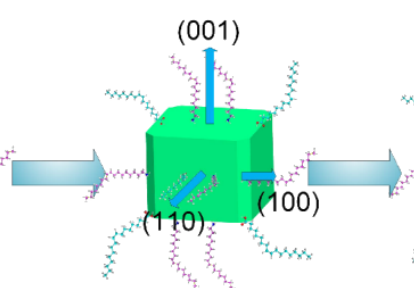

(2)

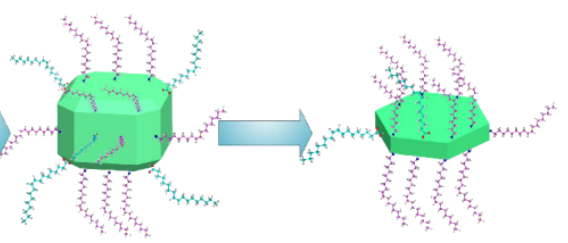

(3)

\footnotetext{
$2+4 y+2+2$ Oleic acid (OA)
}

$x^{2}+x^{2}+5 x$ Oleylamine (OAm)

Figure 5: The change of (a) UV-vis absorption spectra, (b) PL spectra, and (c)-(h) TEM images of $\mathrm{CsPbBr}_{3} \mathrm{PNCs}$ synthesized at $0.5,1.0,1.5,2.0$, 2.5 , and $3.0 \mathrm{~mL}$ of $\mathrm{OAm}$, respectively, while keeping $\mathrm{OA}$ unchanged. (i) Schematic illustration of the morphology change from $\mathrm{CsPbBr}_{3} \mathrm{PNCs}$ to $\mathrm{Cs}_{4} \mathrm{PbBr}_{6} \mathrm{PNCs}$.

$\mathrm{Cs}_{\mathrm{PbBr}}$ to $\mathrm{Cs}_{4} \mathrm{PbBr}_{6}$ leads to a remarkable change in crystal structure and atomic composition. Udayabhaskararao et al. demonstrated that this transformation is driven by recrystallization induced by micelle formation or soft-ligand templating [25]. This mechanism, however, cannot explain the phase transformation from $\mathrm{CsPbBr}_{3}$ to $\mathrm{Cs}_{4} \mathrm{PbBr}_{6}$ in this work because there are no intermediate stages observed by TEM. Therefore, we suppose the transformation between the two phases involves ion equilibria. A large amount of OAm can form oleylammonium and dissolve $\mathrm{PbBr}_{2}$, resulting in the formation of lead oleate and oleylammonium bromide, thus driving the transformation [41]. An even larger amount of OAm can also dissolve $\mathrm{CsPbr}_{3}$ PNCs and accelerate the transformation into $\mathrm{Cs}_{4} \mathrm{PbBr}_{6}$ PNCs. This process is related to Ostwald ripening that was found during the nucleation and growth of PNCs [42]. Therefore, the formation of $\mathrm{Cs}_{4} \mathrm{PbBr}_{6}$ PNCs is promoted by the capacity of the organic ligands to dissolve $\mathrm{PbBr}_{2}$ and by the dissociation of $\mathrm{CsPbBr}_{3}$ PNCs. 
A series of TEM images (Figure $4 c-h$ ) clearly confirm that the morphology of these PNCs can be tuned easily by changing the amount of OAm in the precursor solution, while keeping the amount of OA unchanged. We attribute this morphology change to the crystal anisotropy induced by the growth kinetics. The capping ligands are preferentially attached to the PNCs facets, resulting in different growth rates on different crystal facets [43]. A schematic illustration of the morphology change between $\mathrm{CsPbBr}_{3} \mathrm{PNCs}$ and $\mathrm{Cs}_{4} \mathrm{PbBr}_{6}$ PNCs is shown in Figure $5 \mathrm{i}$. When equal amounts of $\mathrm{OA}$ and $\mathrm{OAm}$ are added, the reaction favors isotropic growth, since OA and OAm play a cooperative role (Figure 5i(1)). When more OAm is added, the long-chain OAm are more easily bound to the surface of the PNCs and restrict the perpendicular growth (001) [44]. Additionally, the growth rates for the side planes are different due to excess amount of OAm easily aggregated at the boundary of two adjacent planes [45], which possibly leads to the formation of (100) planes and the appearance of diamond-like product, as demonstrated in Figure 5i(2,3). This inhibiting effect is distinct when OAm is added in large excess, yielding a hexagonal structure with sharp edges, as shown in Figure 5i(4).

\section{Reversible transformation between $\mathrm{Cs}_{4} \mathrm{PbBr}_{6}$ PNCs and $\mathrm{CsPbBr}_{3}$ PNCs}

After a few weeks, the prepared $\mathrm{Cs}_{4} \mathrm{PbBr}_{6} \mathrm{PNCs}$ solution became milky white, indicating that untransformed $\mathrm{CsPbr}_{3}$ PNCs decomposed completely. We further explored a possible reversible transformation by introducing different amounts of water. When little water was added, the color of $\mathrm{Cs}_{4} \mathrm{PbBr}_{6}$ solution changed from colorless to light-green rapidly (Supporting Information File 2), implying a possible structural transformation. In order to monitor the transformation process, different amounts of water were gradually dropped into a $\mathrm{Cs}_{4} \mathrm{PbBr}_{6}$ PNCs solution (Figure 6a). When more water was added, the solution became green-yellow.

Furthermore, the transformation process was studied by using $\mathrm{UV}$-vis absorption and PL emission spectroscopy. As shown in Figure 6b, the colorless $\mathrm{Cs}_{4} \mathrm{PbBr}_{6} \mathrm{PNCs}$ solution exhibits a strong first excitonic absorption peak at $315 \mathrm{~nm}$. When a small amount of water was slowly added, the intensity of the first excitonic absorption peak declined gradually, indicating the decomposition of $\mathrm{Cs}_{4} \mathrm{PbBr}_{6}$ PNCs. Correspondingly, a weak absorption peak at $510 \mathrm{~nm}$ emerged. Moreover, as the amount of water was increased, the intensity of the absorption peak at about $510 \mathrm{~nm}$ increased steadily (inset in Figure 6b). Compared with the excitonic absorption peak of $\mathrm{Cs}_{4} \mathrm{PbBr}_{6}$ PNCs, the product displayed only weak absorbance. Figure $6 \mathrm{c}$ demonstrates the PL emission spectra of the samples during the transformation process. After the addition of small amounts of water, a PL emission peak at $518 \mathrm{~nm}$ appeared and gradually increased in intensity, suggesting a luminescent product was formed. XRD measurements were carried out to determine the phase of the obtained product. As shown in Figure 6d, the XRD diffraction pattern of final product is consistent with bulk orthorhombic $\mathrm{CsPbr}_{3}$ (PDF card \#18-0364), suggesting the formation of

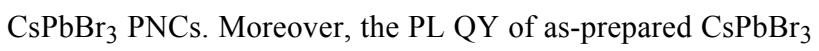
PNCs was calculated to be ca. $70 \%$. Interestingly, the $\mathrm{CsPbr}_{3}$ PNCs show a high stability in ambient environment, as shown in Figure 6e. Upon the addition of a large amount of OAm and upon ultrasonication, the conversion from $\mathrm{CsPbBr}_{3}$ PNCs to $\mathrm{Cs}_{4} \mathrm{PbBr}_{6} \mathrm{PNCs}_{\text {was }}$ achieved (Supporting Information File 1, Figure S3) and can be repeated more than two times, similar to previous reports [25].

The addition of led to the decomposition of $\mathrm{Cs}_{4} \mathrm{PbBr}_{6}$ and the formation of $\mathrm{CsPbBr}_{3}$ PNCs, triggered by the stripping of water-soluble CsBr. During this process, the rhombohedral

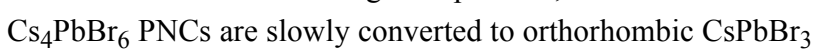
PNCs (Figure 6d) and the rate of this conversion depends on the amount of water. The CsBr-stripping can be proven by the reduction of crystal size from 65 to $11.7 \mathrm{~nm}$ (Figure 1 and Figure 4). This is consistent with the findings reported by $\mathrm{Wu}$ and co-workers [20]. As the solubility of liquid paraffin or capping ligands in water is very low, further dissolution of $\mathrm{CsPbBr}_{3} \mathrm{PNCs}$ is inhibited, which is similar to the effect demonstrated by $\mathrm{Wu}$ 's group who took advantage of the very low (only $9.5 \mathrm{mg} / \mathrm{L}$ ) solubility of hexane in water [20]. The above result indicates that the $\mathrm{CsPbr}_{3}$ PNCs have a higher stability than $\mathrm{Cs}_{4} \mathrm{PbBr}_{6}$ PNCs against water.

\section{Conclusion}

In summary, we demonstrate the effect of small changes in the environment of capping ligands and water on the crystal structure and stoichiometry of PNCs. This study expanded our recent work of synthesizing differently shaped $\mathrm{CsPbBr}_{3}$ PNCs [30]. Similarly as demonstrated in our recent study, by changing the ultrasound power and radiation time, the PL emission of $\mathrm{CsPbBr}_{3} \mathrm{PNCs}$ could be easily tuned. More importantly, with lower the immersion heights of the vibrating spear higher PL $\mathrm{QY}$ of $\mathrm{CsPbBr}_{3}$ PNCs were achieved. The as-prepared $\mathrm{CsPbBr}_{3}$ PNCs show a high PL QY of up to $85 \%$ and a considerable photostability and chemical stability. The $\mathrm{Cs}_{4} \mathrm{PbBr}_{6} \mathrm{PNCs}_{\text {are }}$ obtained via direct ultrasonication of precursors or after adding $\mathrm{OAm}$ in the pre-synthesized $\mathrm{CsPbBr}_{3}$ PNCs solution. The phase transformation of orthorhombic $\mathrm{CsPbr}_{3} \mathrm{NCs}$ to rhombohedral $\mathrm{Cs}_{4} \mathrm{PbBr}_{6} \mathrm{NCs}$ is promoted by the capacity of organic ligands to dissolve $\mathrm{PbBr}_{2}$, and by the formation of lead oleate and the dissociation of $\mathrm{CsPbBr}_{3}$ PNCs. Morphology changes are mainly ascribed to the anisotropic growth of the crystals. In addition, a reverse transformation from $\mathrm{Cs}_{4} \mathrm{PbBr}_{6} \mathrm{PNCs}$ to $\mathrm{Cs}_{2} \mathrm{PbBr}_{3}$ PNCs can be achieved by adding water to pre-synthesized $\mathrm{Cs}_{4} \mathrm{PbBr}_{6}$ 
(a)

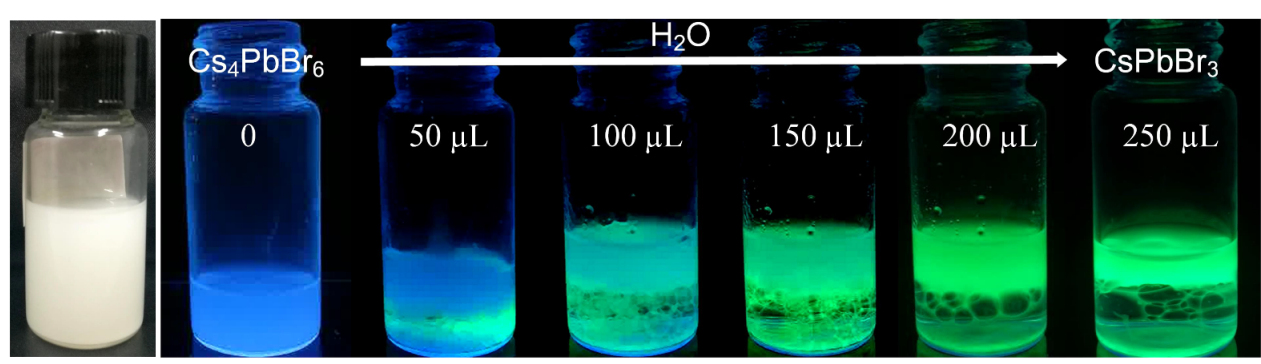

(b)

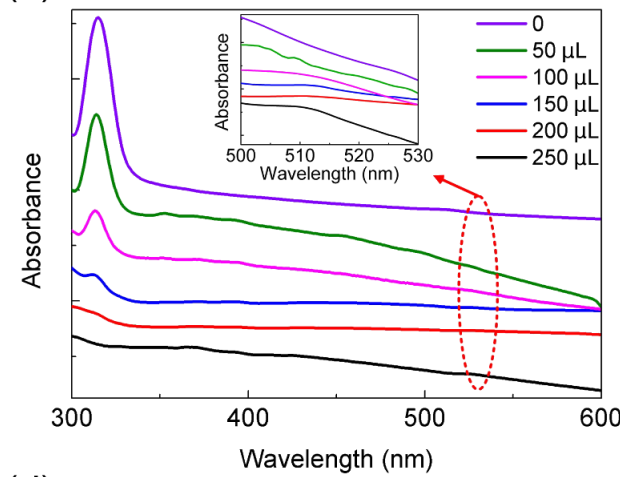

(d)

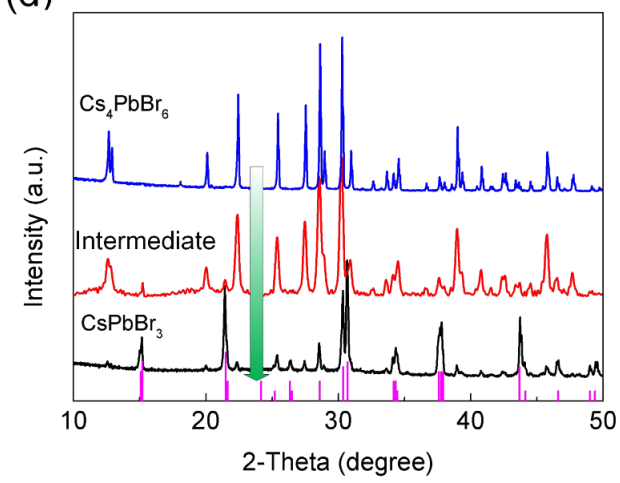

(c)

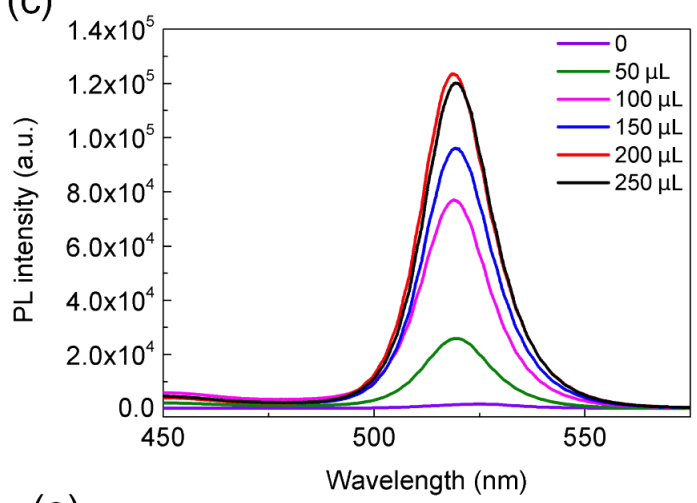

(e)

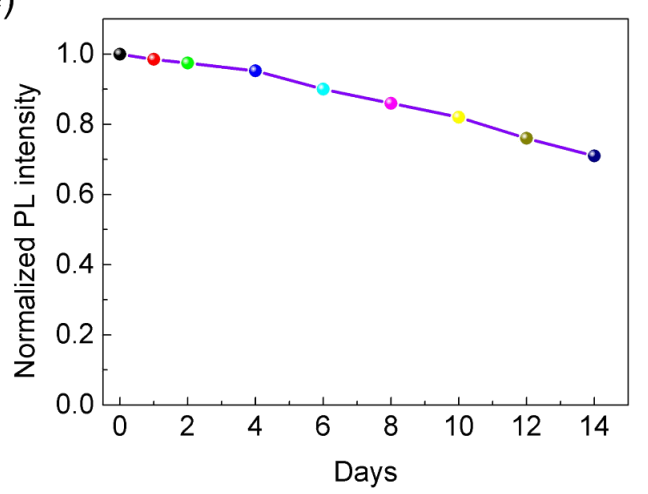

Figure 6: (a) The transformation process from $\mathrm{Cs}_{4} \mathrm{PbBr}_{6} \mathrm{PNCs}$ to $\mathrm{CsPbBr}_{3} \mathrm{PNCs}$. The products were illuminated under a $365 \mathrm{~nm}$ UV light. Both (b) UV-vis absorption spectra and (c) PL spectra of products were recorded during the transformation process. (d) XRD diffraction patterns of typical products prepared by changing the amount of added water, demonstrating the transformation from rhombohedral $\mathrm{Cs}_{4} \mathrm{PbBr}_{6} \mathrm{PNCs}_{5}$ to orthorhombic $\mathrm{CsPbBr}_{3}$ PNCs. (e) Stability of as-prepared $\mathrm{CsPbBr}_{3}$ PNCs in ambient environment.

PNCs. The developed ultrasonication assistance results in the successful control over the phase transformation of PNCs, which can find widespread application in photoelectronic devices. We anticipate that this work can be extended to prepare other halide perovskites.

\section{Experimental}

\section{Chemicals}

Cesium carbonate $\left(\mathrm{Cs}_{2} \mathrm{CO}_{3}, 99 \%\right)$, lead bromide $\left(\mathrm{PbBr}_{2}, 98 \%\right)$, liquid paraffin (90\%), oleic acid (OA, 90\%), oleylamine (OAm, $70 \%$ ), and anhydrous toluene $(99.8 \%)$ were purchased from Shanghai Aladdin Biochemical Technology Co. The chemicals used in the present work were of analytical grade and used without further purifications.

\section{Synthesis of $\mathrm{CsPbBr}_{3}$ PNCs and $\mathrm{Cs}_{4} \mathrm{PbBr}_{6}$ PNCs}

$\mathrm{CsPbBr}_{3}$ PNCs: The PNCs were prepared via modifying the procedures reported by Tong and Rao and co-workers [29,30]. In a typical process, $\mathrm{Cs}_{2} \mathrm{CO}_{3}(0.15 \mathrm{mmol})$ and $\mathrm{PbBr}_{2}$ $(0.30 \mathrm{mmol})$ powders were added to a mixture of $10 \mathrm{~mL}$ liquid paraffin (LP), $0.50 \mathrm{~mL} \mathrm{OA}$ and $0.50 \mathrm{~mL}$ OAm. Then the reaction medium was processed by tip-sonication at a power of $120 \mathrm{~W}$ for $40 \mathrm{~min}$. During the sonication, the colorless reaction medium gradually transformed into a yellow and then an orange-yellow solution, which suggests the formation of PNCs and demonstrates strong fluorescence emission under $365 \mathrm{~nm}$ UV light excitation. After completion of the reaction, unreacted precursors and excess ligands were removed by centrifugation 
at a speed of $3000 \mathrm{rpm}$ for $10 \mathrm{~min}$ and then the precipitate was redispersed in $5.0 \mathrm{~mL}$ of toluene. Then, the colloidal solution was centrifuged at a speed of $12000 \mathrm{rpm}$ for $5 \mathrm{~min}$ and the sediment was redispersed in toluene for further characterization.

$\mathbf{C s}_{4} \mathbf{P b B r}_{6}$ PNCs: $\mathrm{Cs}_{2} \mathrm{CO}_{3}(0.15 \mathrm{mmol})$ and $\mathrm{PbBr}_{2}(0.30 \mathrm{mmol})$ powders were added to a mixture of $10 \mathrm{~mL}$ liquid paraffin, $0.50 \mathrm{~mL} \mathrm{OA}$ and $3.0 \mathrm{~mL} \mathrm{OAm}$, while keeping other synthesis conditions as the same as that of $\mathrm{CsPbrr}_{3}$ PNCs.

\section{Reversible transformation from $\mathrm{Cs}_{4} \mathrm{PbBr}_{6}$ PNCs to $\mathrm{CsPbBr}_{3}$ PNCs}

$50-250 \mu \mathrm{L}$ of water was added to $5.0 \mathrm{~mL}$ of the pre-synthesized $\mathrm{Cs}_{4} \mathrm{PbBr}_{6} \mathrm{PNCs}$ solution and shaken slightly, which is a modification of the work reported by $\mathrm{Wu}$ and co-workers [20].

\section{Characterizations}

The crystal surface morphology of the PNCs was characterized by transmission electron microscopy (TEM, JEM-2100F, JEOL, Japan) with an accelerating voltage of $100 \mathrm{kV}$. High-resolution TEM (HRTEM) was carried out on a JEOL JEM-2100F instrument operating at $200 \mathrm{kV}$. The crystal phases of the products were measured using an X-ray diffractometer (XRD, D8-Advance, Bruker, Germany) with a $\mathrm{Cu} \mathrm{K} \alpha$ radiation source $(\lambda=0.15418 \mathrm{~nm})$ at a counting rate of $2^{\circ}$ per minute in the scanning angle $(2 \theta)$ range from $5^{\circ}$ to $50^{\circ}$. The surface morphology of $\mathrm{Cs}_{4} \mathrm{PbBr}_{6}$ PNCs was characterized by using a field-emission scanning electron microscope (SEM, Merlin). The UV-vis absorption spectra of the samples were measured using a UV-vis spectrometer (Shimadzu, Japan) over the wavelength range from 300 to $700 \mathrm{~nm}$, at $1 \mathrm{~nm}$ intervals. The PL spectra of the PNCs were recorded using a fluorescence spectrophotometer (RF-6000, Shimadzu, Japan) using a Xe lamp as an excitation source.

\section{Supporting Information}

\section{Supporting Information File 1}

Additional PL spectra, SEM image, and UV-vis absorption spectra.

[https://www.beilstein-journals.org/bjnano/content/ supplementary/2190-4286-10-66-S1.pdf]

\section{Supporting Information File 2}

Video showing the transformation from $\mathrm{Cs}_{4} \mathrm{PbBr}_{6}$ to $\mathrm{CsPbBr}_{3} \mathrm{PNCs}$ after addition of water.

[https://www.beilstein-journals.org/bjnano/content/ supplementary/2190-4286-10-66-S2.mp4]

\section{Acknowledgements}

This work is financially supported by National Natural Science Foundation of China (51735004, 51805173, and 51775199), Natural Science Foundation of Guangdong Province (2014A030312017), Science \& Technology Program of Guangdong Province (2017B010115001), and the SCUT Fundamental Research Funds for the Central Universities.

\section{ORCID ${ }^{\circledR} \mathrm{iDs}$}

Jin Z. Zhang - https://orcid.org/0000-0003-3437-912X

\section{References}

1. Pan, J.; Quan, L. N.; Zhao, Y.; Peng, W.; Murali, B.; Sarmah, S. P.; Yuan, M.; Sinatra, L.; Alyami, N. M.; Liu, J.; Yassitepe, E.; Yang, Z.; Voznyy, O.; Comin, R.; Hedhili, M. N.; Mohammed, O. F.; Lu, Z. H.; Kim, D. H.; Sargent, E. H.; Bakr, O. M. Adv. Mater. (Weinheim, Ger.) 2016, 28, 8718-8725. doi:10.1002/adma.201600784

2. Li, J.; Xu, L.; Wang, T.; Song, J.; Chen, J.; Xue, J.; Dong, Y.; Cai, B.; Shan, Q.; Han, B.; Zeng, H. Adv. Mater. (Weinheim, Ger.) 2017, 29, 1603885. doi:10.1002/adma.201603885

3. Jeon, N. J.; Na, H.; Jung, E. H.; Yang, T.-Y.; Lee, Y. G.; Kim, G.; Shin, H.-W.; II Seok, S.; Lee, J.; Seo, J. Nat. Energy 2018, 3, 682-689. doi:10.1038/s41560-018-0200-6

4. Wang, X.; Shoaib, M.; Wang, X.; Zhang, X.; He, M.; Luo, Z.; Zheng, W.; Li, H.; Yang, T.; Zhu, X.; Ma, L.; Pan, A. ACS Nano 2018, 12, 6170-6178. doi:10.1021/acsnano.8b02793

5. Yang, T.; Zheng, Y.; Du, Z.; Liu, W.; Yang, Z.; Gao, F.; Wang, L.; Chou, K.-C.; Hou, X.; Yang, W. ACS Nano 2018, 12, 1611-1617. doi:10.1021/acsnano.7b08201

6. Vickers, E. T.; Graham, T. A.; Chowdhury, A. H.; Bahrami, B.; Dreskin, B. W.; Lindley, S.; Bonabi Naghadeh, S.; Qiao, Q.; Zhang, J. Z. ACS Energy Lett. 2018, 3, 2931-2939. doi:10.1021/acsenergylett.8b01754

7. Koscher, B. A.; Swabeck, J. K.; Bronstein, N. D.; Alivisatos, A. P. J. Am. Chem. Soc. 2017, 139, 6566-6569. doi:10.1021/jacs.7b02817

8. Wu, K.; Liang, G.; Shang, Q.; Ren, Y.; Kong, D.; Lian, T. J. Am. Chem. Soc. 2015, 137, 12792-12795. doi:10.1021/jacs.5b08520

9. Akkerman, Q. A.; Park, S.; Radicchi, E.; Nunzi, F.; Mosconi, E.; De Angelis, F.; Brescia, R.; Rastogi, P.; Prato, M.; Manna, L. Nano Lett. 2017, 17, 1924-1930. doi:10.1021/acs.nanolett.6b05262

10. Yin, J.; Zhang, Y.; Bruno, A.; Soci, C.; Bakr, O. M.; Brédas, J.-L.; Mohammed, O. F. ACS Energy Lett. 2017, 2, 2805-2811. doi:10.1021/acsenergylett.7b01026

11. Yin, J.; Maity, P.; De Bastiani, M.; Dursun, I.; Bakr, O. M.; Brédas, J.-L.; Mohammed, O. F. Sci. Adv. 2017, 3, e1701793. doi:10.1126/sciadv.1701793

12. Saidaminov, M. I.; Almutlaq, J.; Sarmah, S.; Dursun, I.; Zhumekenov, A. A.; Begum, R.; Pan, J.; Cho, N.; Mohammed, O. F.; Bakr, O. M. ACS Energy Lett. 2016, 1, 840-845. doi:10.1021/acsenergylett.6b00396

13. Seth, S.; Samanta, A. J. Phys. Chem. Lett. 2017, 8, 4461-4467. doi:10.1021/acs.jpclett.7b02100

14. Protesescu, L.; Yakunin, S.; Bodnarchuk, M. I.; Krieg, F.; Caputo, R.; Hendon, C. H.; Yang, R. X.; Walsh, A.; Kovalenko, M. V. Nano Lett. 2015, 15, 3692-3696. doi:10.1021/nl5048779

15. Pan, A.; He, B.; Fan, X.; Liu, Z.; Urban, J. J.; Alivisatos, A. P.; He, L.; Liu, Y. ACS Nano 2016, 10, 7943-7954. doi:10.1021/acsnano.6b03863 
16. He, X.; Quu, Y.; Yang, S. Adv. Mater. (Weinheim, Ger.) 2017, 29 , 1700775. doi:10.1002/adma.201700775

17. Bekenstein, Y.; Koscher, B. A.; Eaton, S. W.; Yang, P.; Alivisatos, A. P. J. Am. Chem. Soc. 2015, 137, 16008-16011. doi:10.1021/jacs.5b11199

18. Chen, M.; Zou, Y.; Wu, L.; Pan, Q.; Yang, D.; Hu, H.; Tan, Y.; Zhong, Q.; Xu, Y.; Liu, H.; Sun, B.; Zhang, Q. Adv. Funct. Mater. 2017, 27, 1701121. doi:10.1002/adfm.201701121

19. Rao, L.; Tang, Y.; Yan, C.; Li, J.; Zhong, G.; Tang, K.; Yu, B.; Li, Z.; Zhang, J. Z. J. Mater. Chem. C 2018, 6, 5375-5383. doi:10.1039/c8tc00582f

20. Wu, L.; Hu, H.; Xu, Y.; Jiang, S.; Chen, M.; Zhong, Q.; Yang, D.; Liu, Q.; Zhao, Y.; Sun, B.; Zhang, Q.; Yin, Y. Nano Lett. 2017, 17, 5799-5804. doi:10.1021/acs.nanolett.7b02896

21. Palazon, F.; Urso, C.; De Trizio, L.; Akkerman, Q.; Marras, S.; Locardi, F.; Nelli, I.; Ferretti, M.; Prato, M.; Manna, L. ACS Energy Lett. 2017, 2, 2445-2448. doi:10.1021/acsenergylett.7b00842

22. Palazon, F.; Almeida, G.; Akkerman, Q. A.; De Trizio, L.; Dang, Z.; Prato, M.; Manna, L. Chem. Mater. 2017, 29, 4167-4171. doi:10.1021/acs.chemmater.7b00895

23. Zhai, W.; Lin, J.; Li, Q.; Zheng, K.; Huang, Y.; Yao, Y.; He, X.; Li, L.; Yu, C.; Liu, C.; Fang, Y.; Liu, Z.; Tang, C. Chem. Mater. 2018, 30, 3714-3721. doi:10.1021/acs.chemmater.8b00612

24. Liu, Z.; Bekenstein, Y.; Ye, X.; Nguyen, S. C.; Swabeck, J.; Zhang, D.; Lee, S.-T.; Yang, P.; Ma, W.; Alivisatos, A. P. J. Am. Chem. Soc. 2017, 139, 5309-5312. doi:10.1021/jacs.7b01409

25. Udayabhaskararao, T.; Houben, L.; Cohen, H.; Menahem, M.; Pinkas, I.; Avram, L.; Wolf, T.; Teitelboim, A.; Leskes, M.; Yaffe, O.; Oron, D.; Kazes, M. Chem. Mater. 2018, 30, 84-93. doi:10.1021/acs.chemmater.7b02425

26. Huang, H.; Xue, Q.; Chen, B.; Xiong, Y.; Schneider, J.; Zhi, C.; Zhong, H.; Rogach, A. L. Angew. Chem. 2017, 129, 9699-9704. doi:10.1002/ange.201705595

27. Fang, F.; Chen, W.; Li, Y.; Liu, H.; Mei, M.; Zhang, R.; Hao, J.; Mikita, M.; Cao, W.; Pan, R.; Wang, K.; Sun, X. W. Adv. Funct. Mater. 2018, 28, 1706000. doi:10.1002/adfm.201706000

28. Li, X.; Yu, D.; Cao, F.; Gu, Y.; Wei, Y.; Wu, Y.; Song, J.; Zeng, H. Adv. Funct. Mater. 2016, 26, 5903-5912. doi:10.1002/adfm.201601571

29. Tong, Y.; Bladt, E.; Aygüler, M. F.; Manzi, A.; Milowska, K. Z.; Hintermayr, V. A.; Docampo, P.; Bals, S.; Urban, A. S.; Polavarapu, L.; Feldmann, J. Angew. Chem., Int. Ed. 2016, 55, 13887-13892. doi:10.1002/anie.201605909

30. Rao, L.; Tang, Y.; Song, C.; Xu, K.; Vickers, E. T.; Bonabi Naghadeh, S.; Ding, X.; Li, Z.; Zhang, J. Z. Chem. Mater. 2019, 31, 365-375. doi:10.1021/acs.chemmater.8b03298

31. Brouwer, A. M. Pure Appl. Chem. 2011, 83, 2213-2228. doi:10.1351/pac-rep-10-09-31

32. Qi, X.; Zhou, T.; Deng, S.; Zong, G.; Yao, X.; Fu, Q. J. Mater. Sci. 2014, 49, 1785-1793. doi:10.1007/s10853-013-7866-8

33. Mphuthi, N. G.; Adekunle, A. S.; Fayemi, O. E.; Olasunkanmi, L. O.; Ebenso, E. E. Sci. Rep. 2017, 7, 43181. doi:10.1038/srep43181

34. Yuwen, L.; Yu, H.; Yang, X.; Zhou, J.; Zhang, Q.; Zhang, Y.; Luo, Z.; Su, S.; Wang, L. Chem. Commun. 2016, 52, 529-532. doi:10.1039/c5cc07301d

35. Akkerman, Q. A.; Motti, S. G.; Srimath Kandada, A. R.; Mosconi, E.; D'Innocenzo, V.; Bertoni, G.; Marras, S.; Kamino, B. A.; Miranda, L.; De Angelis, F.; Petrozza, A.; Prato, M.; Manna, L. J. Am. Chem. Soc. 2016, 138, 1010-1016. doi:10.1021/jacs.5b12124
36. Dang, Z.; Shamsi, J.; Palazon, F.; Imran, M.; Akkerman, Q. A.; Park, S.; Bertoni, G.; Prato, M.; Brescia, R.; Manna, L. ACS Nano 2017, 11, 2124-2132. doi:10.1021/acsnano.6b08324

37. Seth, S.; Samanta, A. J. Phys. Chem. Lett. 2018, 9, 176-183. doi:10.1021/acs.jpclett.7b02931

38. Akkerman, Q. A.; Abdelhady, A. L.; Manna, L. J. Phys. Chem. Lett. 2018, 9, 2326-2337. doi:10.1021/acs.jpclett.8b00572

39. Zhang, Y.; Saidaminov, M. I.; Dursun, I.; Yang, H.; Murali, B.; Alarousu, E.; Yengel, E.; Alshankiti, B. A.; Bakr, O. M.; Mohammed, O. F. J. Phys. Chem. Lett. 2017, 8, 961-965. doi:10.1021/acs.jpclett.7b00105

40. Chen, D.; Wan, Z.; Chen, X.; Yuan, Y.; Zhong, J. J. Mater. Chem. C 2016, 4, 10646-10653. doi:10.1039/c6tc04036e

41. Almeida, G.; Goldoni, L.; Akkerman, Q.; Dang, Z.; Khan, A. H.; Marras, S.; Moreels, I.; Manna, L. ACS Nano 2018, 12, 1704-1711. doi:10.1021/acsnano.7b08357

42. Koolyk, M.; Amgar, D.; Aharon, S.; Etgar, L. Nanoscale 2016, 8 , 6403-6409. doi:10.1039/c5nr09127f

43. Liang, Z.; Zhao, S.; Xu, Z.; Qiao, B.; Song, P.; Gao, D.; Xu, X. ACS Appl. Mater. Interfaces 2016, 8, 28824-28830. doi:10.1021/acsami.6b08528

44. Liu, W.; Zheng, J.; Cao, S.; Wang, L.; Gao, F.; Chou, K.-C.; Hou, X.; Yang, W. Inorg. Chem. 2018, 57, 1598-1603. doi:10.1021/acs.inorgchem.7b02941

45. Li, G.; Wang, H.; Zhu, Z.; Chang, Y.; Zhang, T.; Song, Z.; Jiang, Y. Chem. Commun. 2016, 52, 11296-11299. doi:10.1039/c6cc05877a

\section{License and Terms}

This is an Open Access article under the terms of the Creative Commons Attribution License (http://creativecommons.org/licenses/by/4.0). Please note that the reuse, redistribution and reproduction in particular requires that the authors and source are credited.

The license is subject to the Beilstein Journal of Nanotechnology terms and conditions: (https://www.beilstein-journals.org/bjnano)

The definitive version of this article is the electronic one which can be found at: $\underline{\text { doi:10.3762/bjnano. } 10.66}$ 